CASE REPORT

Turkish Journal of Geriatrics

DOI: 10.31086/tjgeri.2020.163

2020; 23(2): 278-282

- Adem SANCl ${ }^{1}$

- Basak GULPINAR² (D)

CORRESPONDANCE

Adem SANCI

Ankara University, Faculty of Medicine, Ibni

Sina Hospital, UROLOGY Department, Ankara,

TURKEY.

Phone: +905349281130

e-mail: dr.adem88@hotmail.com

Received: February 22, 2020

Accepted: April 20, 2020

Ankara University Faculty of Medicine, Ibni Sina Hospital, UROLOGY Department,

Ankara, TURKEY.

${ }^{2}$ Ankara University Faculty of Medicine, Ibni Sina Hospital, RADIOLOGY Department,

Ankara, TURKEY.

\section{IMMUNOGLOBULIN G4-RELATED DISEASE PRESENTING AS URETERAL MALIGNANCY AND URETERAL STRICTURE TREATED WITH AZATHIOPRINE AFTER SURGERY IN A GERIATRIC PATIENT WITH A SINGLE FUNCTIONAL KIDNEY: A CASE REPORT}

\begin{abstract}
Immunoglobulin G4-related sclerosing ureteral disease is a rare benign disorder characterised by fibrosis and lymphoplasmacytic infiltration in the ureter. A 70-year-old man with a single functional kidney and left flank pain was diagnosed with IgG4-related ureteral disease that presented as a unilateral ureteral mass. Left hydronephrosis and a $25 \times 23 \times 26 \mathrm{~mm}$ left midureteral mass were found. No malignancy was found on ureteroscopy and urinary cytology did not reveal any neoplastic cells. A $2 \mathrm{~cm}$ midureteral stenosis was found in the left ureter on retrograde pyelography. It was not a ureteral stricture but was the result of periureteral inflammation and fibrosis caused by immunoglobulin G4-related sclerosing disease. Initial endoscopic ablation-obliteration therapy was unsuccessful, and after 6 weeks the patient was treated by robotic ureteroureterostomy. Most plasma cells in the excised ureteral segment were IgG4-positive. Serum IgG4 was $273 \mathrm{mg} / \mathrm{dL}$ (normal range: $85-120 \mathrm{mg} / \mathrm{dL}$ ). The histology of the ureteral segment resembled retroperitoneal fibrosis and the histopathology of the stricture included IgG4-positive cells, fibrosis and ureteritis. The patient was treated with oral azathioprine for 6 months. No evidence of recurrence was seen on ureteroscopy or abdominopelvic computed tomography at the 3-month or 1-year follow-up.
\end{abstract}

Keywords: Robotics; Immunoglobulins; Ureter; Neoplasms. 


\section{INTRODUCTION}

Ureteral stricture is an uncommon benign disorder that may cause acute renal failure if untreated. Most are iatrogenic, but they can also result from infection, endoscopic procedures, trauma, radiotherapy, pelvic surgery, retroperitoneal fibrosis, malignancy, or immunoglobulin G4related ureteral disease (IgG4-RUD). IgG4-RUD is a rare condition characterised by fibrosis and lymphoplasmacytic infiltration of the ureter. Only 15 cases have been reported. (1). The most common ureteral manifestation of IgG4-RUD is ureteritis. Mass-forming lesions, segmental stenosis, extrinsic narrowing of the ureter, or ureteral strictures may also occur. The surgical management of ureteral strictures depends the location and length and include open, laparoscopic, or robotic ureteroureterostomy; ureteral reimplantation, or nephrectomy. Other endourological treatments include balloon dilation, cold knife incision, ablation-obliteration therapy, and laser endoureterotomy (2). This IgG4-RUD patient presented with a ureteral massforming lesion that was treated by robot-assisted surgery.

\section{CASE REPORT}

A 70-year-old man presented with left flank pain. The patient had a single functioning kidney. The right kidney was dysfunctional because of urolithiasis and previous surgeries for stone disease. Ultrasonography findings were right grade 2 hydronephrosis, right atrophic kidney, left grade 4 hydronephrosis and a left midureteral mass suspicious for ureteral cancer. Magnetic resonance imaging (MRI) revealed a $25 \times 23 \times 26 \mathrm{~mm}$ left midureteral mass, left grade 4 hydronephrosis, right grade 3 hydronephrosis, and a atrophic right kidney (Fig. 1).

Figure 1. Solid mass in the middle-distal left ureter at the level of iliac bifurcation; the lesion was hypointense on T2-weighted images (A) and strongly enhanced on fat-suppressed, T1-weighted contrast-enhanced images (B) with grade 4 hydronephrosis.

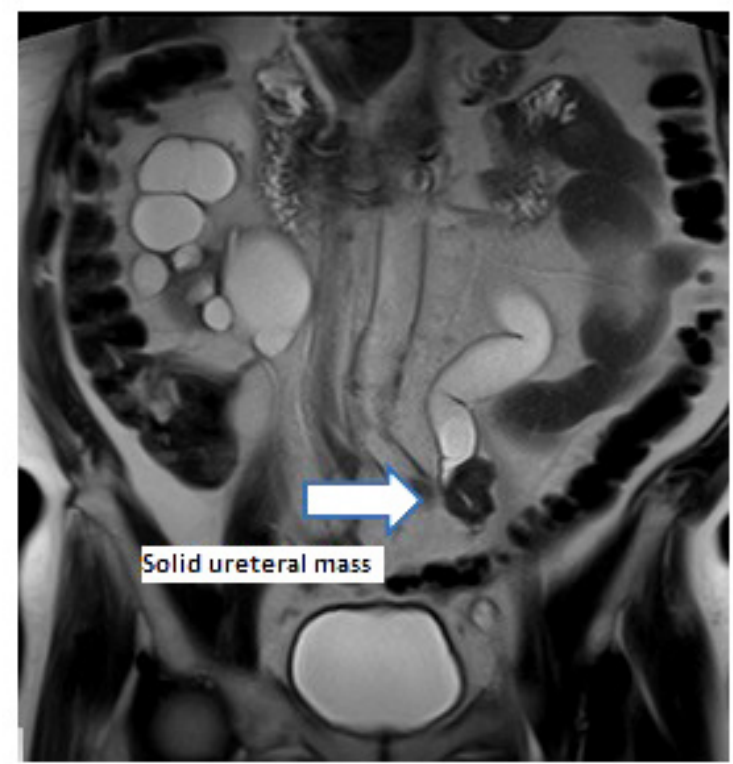

A

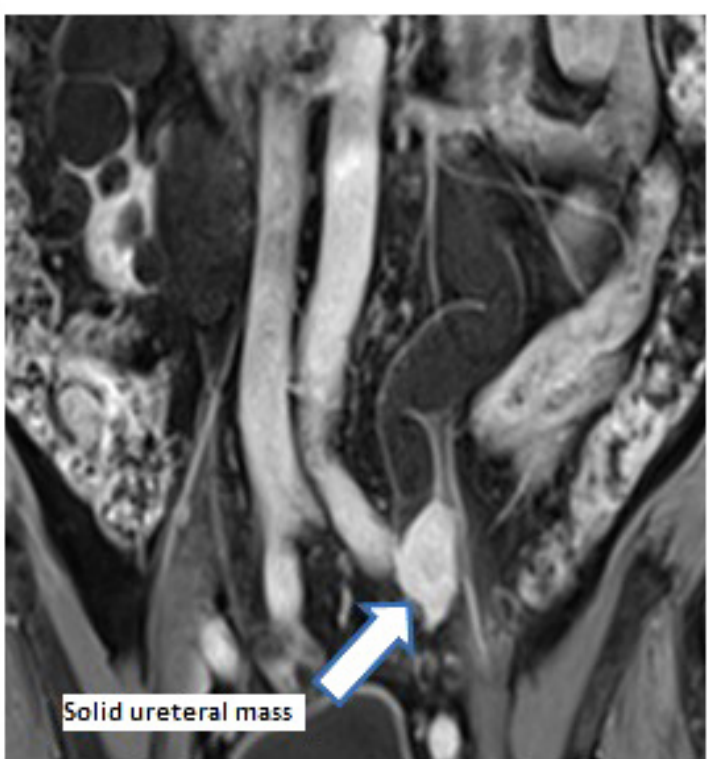

B 
The MRI contrast agent did not affect the patient's renal function. T2-weighted MRI was used instead of computed tomography (CT) because it has been reported to be more useful for demonstrating dilated or obstructed collecting systems (3). The hydronephrosis in the right kidney was chronic and resulted from stone disease. The hydronephrosis in the left kidney was newly developed and caused by the ureteral mass. Renal function evaluated by diuretic renal scintigraphy using a standard protocol was $81 \%$ for the left and $19 \%$ for the right kidney. Treatment for hydronephrosis in the right atrophic kidney was not considered because it was asymptomatic. Retrograde pyelography (RPG) and diagnostic ureteroscopy (URS) were performed before nephroureterectomy of the mass in the left ureter because the patient had a single functioning kidney. A $2 \mathrm{~cm}$ left midureteral stricture was found. No malignant lesions were detected, but a mass causing extrinsic narrowing of the ureter was found. A biopsy revealed only nonspecific ureteritis and increased fibrosis; urinary cytology did not reveal any neoplastic cells. Endoscopic obliteration therapy was performed. A 6-Fr, $26-\mathrm{cm}$ double-J stent was implanted and was cystoscopically removed 6 weeks later. The mass was large for successful endoscopic obliteration, but that treatment was performed to avoid both unnecessary nephroureterectomy and permanent dialysis. A random interventional radiologyguided biopsy on day 3 after the procedure revealed fibrosis, abundant lgG4-positive cells and ureteritis that confirmed the diagnosis. When the catheter was removed 6 weeks after obliteration therapy, it was found that creatinine progression had occurred and the anteroposterior diameter of the renal pelvis had increased.

The patient was treated by robotic ureteroureterostomy for ureteral stricture caused by ureteral IgG4 sclerosing disease. The operative time was 193 minutes, the console time was 112 minutes, and the intraoperative blood loss was approximately $50 \mathrm{~mL}$. Blood transfusion was not required. The drainage catheter was removed on the second postoperative day, and the double-J catheter was removed cystoscopically 6 weeks later. The patient was discharged on postoperative day 2. The histopathological findings of the urethral stricture included abundant lgG4-positive cells, fibrosis, and ureteritis (Figures 2 and 3).

Figure 2. Segmental resection of the ureter (A), ureteral stricture caused by periureteral inflammation and fibrosis (B).
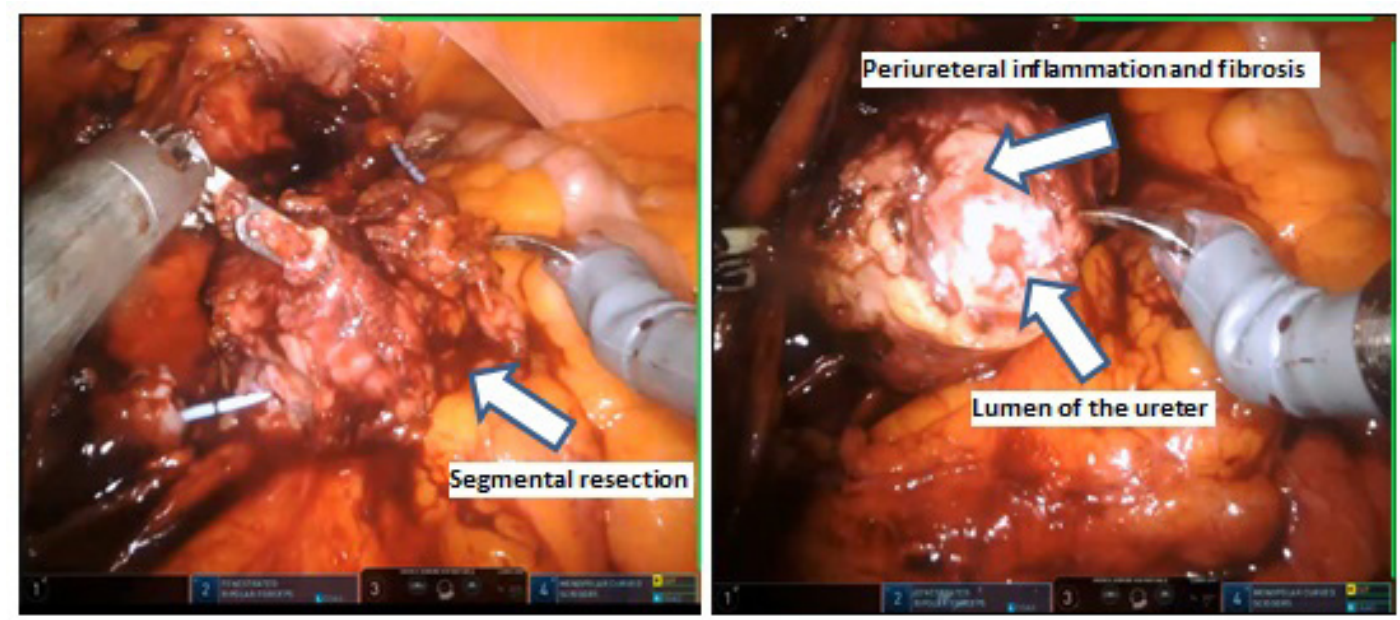
Figure 3. Microscopic scans of the immunoglobulin G4-related ureteral disease of the ureter. Aggregated IGG4-positive plasma cells (A), Overly increased fibrosis and prominent inflammatory cells (B).

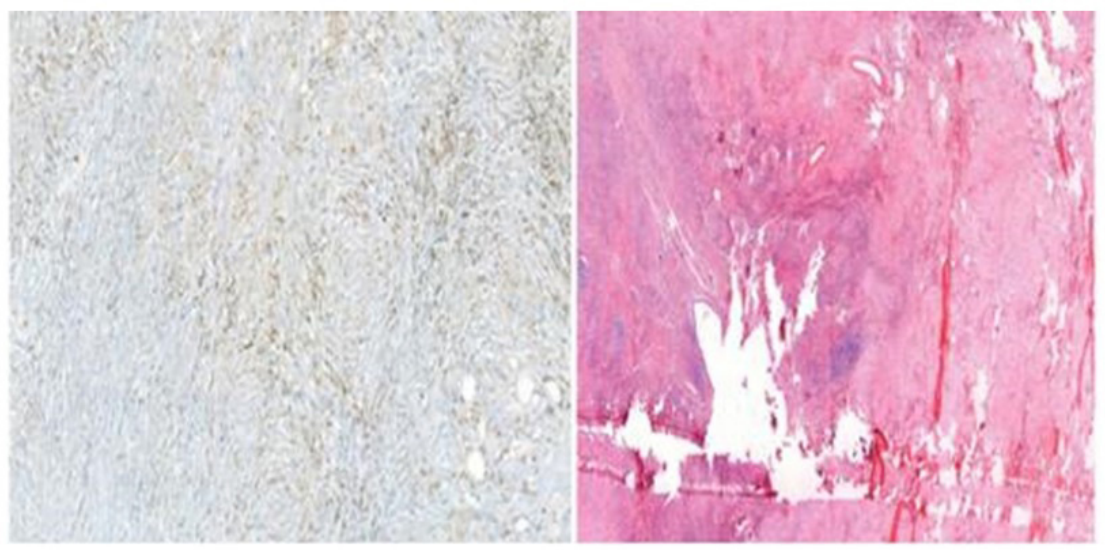

A

The patient's blood lgG4 concentration was $273 \mathrm{mg} / \mathrm{dL}$ (normal range: $85-120 \mathrm{mg} / \mathrm{dL}$ ) and he was treated with oral azathioprine for 6 months by the rheumatology department. At the 3-month follow-up, ultrasonography revealed grade 1 left hydronephrosis. The flank pain had subsided, and the creatinine had decreased from 2.15 to 1.35 $\mathrm{mg} / \mathrm{dL}$. No evidence of recurrence was seen on URS and abdominopelvic CT at 3 months and 1 year after discharge.

\section{DISCUSSION}

IgG4-RUD is a rare inflammatory disorder that can mimic mass-forming lesions. It is characterised by a lymphoplasmacytic infiltrate abundant in IgG4-positive plasma cells and increased fibrosis. Serum IgG4 may be elevated (4). Immunoglobulin G4-related disease (IgG4-RD) can affect any organ or system, including the pancreas, thyroid, salivary glands, or ureter (5). Although IgG4-RD of the urinary tract usually involves the urinary bladder, it can occur in the kidneys, prostate, or ureter (6). Radiological diagnosis of ureteral involvement may be challenging if malignancy- mimicking lesions are present. In such cases, the presence of biochemical, clinical or pathological abnormalities in addition to the radiological evidence is helpful. The most common ureteral manifestation is ureteritis followed by massforming lesions, segmental stenosis or stricture and extraureteral lesions with secondary ureteral involvement. IgG4-RD of the ureter may rarely present as hydronephrosis arising in the ureter and lead to acute kidney injury with spontaneous remission (7). IgG4-RD of the ureter is diagnosed by pathological, radiological and ureteroscopic evaluation and if urine cytology and ureteroscopic biopsy find no evidence of malignancy, this kind of inflammatory lesion should be included in the differential diagnosis. Aggressive treatment, such as nephroureterectomy, should be avoided. An accurate diagnosis is important because antiinflammatory therapy is indicated to prevent recurrent strictures, but no consensus exists as to whether steroids or other medical treatment should be given to patients after resecting the lesion. No specific treatments for IgG4-RD, especially IgG4-RUD, are widely recommended. 
Treatment of IgG4-RD includes a watch-and-wait approach, glucocorticoids, rituximab, azathioprine and steroid-sparing immunomodulators. It has been reported that relapse may occur in $25 \%$ to $50 \%$ of patients after discontinuation of medical treatment (6). The most commonly used nonendoscopic surgery for the treatment of ureteral stricture is ureteroureterostomy, which can be performed with open, laparoscopic or robotassisted procedures. Even though additional evidence is needed for validation, it is our experience that in geriatric patients, robotic surgery has advantages including less analgesia for postoperative pain, less bleeding and a shorter hospital stay compared with other approaches. Its most important disadvantage is a longer procedure time. To our knowledge, this is the first report of a robot-assisted ureteroureterostomy for IgG4-RUD in a geriatric patient.

In conclusion, IgG4-RUD may originate as hydronephrosis in the renal pelvis and ureter,

\section{REFERENCES}

1. Marando A, D'Ambrosio G, Catanzaro F, La Rosa S, Sessa F. IgG4-related disease of the ureter: report of two cases and review of the literature. Virchows Arch 2013;462(6):673-678. (PMID:23666067).

2. Tran H, Arsovska O, Paterson RF, Chew BH. Evaluation of risk factors and treatment options in patients with ureteral stricture disease at a single institution. Can Urol Assoc J 2015;9(11-12):E921-4. (PMID:26788241).

3. Leyendecker JR, Barnes CE, Zagoria RJ. MR Urography: techniques and clinical applications. RadioGraphics 2008;28(1):23-46; discussion 46-7. (PMID:18203929).

4. Abe H, Morikawa T, Araki A, et al. IgG4-related periureteral fibrosis presenting as a unilateral ureteral mass. Pathol Res Pract 2011;207(11):712-4. mimicking a ureteral tumour. Early diagnosis is important because most patients respond to immunosuppressive drugs. To avoid unnecessary nephroureterectomy, diagnostic URS and biopsy are recommended to rule out malignancy, and systemic medical treatment is recommended to prevent recurrence after surgery. Robotic ureteroureterostomy may be a suitable, feasible, effective, and minimally invasive alternative for the treatment of ureteral strictures due IgG4-RUD in cases in which endoscopic treatment has failed. Our experience with this patient adds to what is known about the radiological-pathological diagnosis and surgical-medical treatment outcomes of IgG4RUD. Clinical trials are needed to achieve a better understanding of clinical manifestations, diagnosis and treatment options of patients with IgG4-RUD.

\section{CONFLICT OF INTEREST}

No conflict of interest.
(PMID:21925801).

5. Yu KH, Chan TM, Tsai PH, Chen CH, Chang PY. Diagnostic performance of serum lgG4 levels in patients with IgG4-related disease. Medicine (Baltimore) 2015;94(41):e1707. (PMID:26469909).

6. Harik LR, Merino C, Coindre JM, Amin MB, Pedeutour F, Weiss SW. Pseudosarcomatous myofibroblastic proliferations of the bladder: a clinicopathologic study of 42 cases. Am J Surg Pathol 2006;30(7):78794. (PMID:16819319).

7. Lei WH, Xin J, Shao CX, et al. IgG4 related kidney disease mimicking malignant ureter tumor: case report and literature review. Medicine (Baltimore) 2016;95(3):e2550. (PMID:26817905). 